\title{
Peripheral Extracorporeal Membrane Oxygenation is a safe and effective support modality for pediatric patients with sepsis
}

Brielle Warnock ${ }^{1}$; Joshua Brown ${ }^{1}$; Eamaan Turk; Gail Hocutt RNC ${ }^{2}$; and Brian Gray, MD ${ }^{2}$ ${ }^{1}$ Indiana University School of Medicine, ${ }^{2}$ Riley Hospital for Children, Department of Pediatric Surgery

\section{Background}

Extracorporeal Membrane Oxygenation (ECMO) can be used as a treatment modality for pediatric patients with refractory septic shock. Previous studies indicate central ECMO, with direct cardiac cannulation, is superior for septic patients. At Riley Hospital for Children, we believe that peripheral ECMO support, through cervical or femoral vessels, is an effective and safe method of supporting pediatric septic patients in a less invasive manner.

\section{Methods}

We reviewed pediatric (30 days to 18 years) patients supported with ECMO for septic shock from 2005-2019 at Riley Hospital for Children and compared them to non-septic respiratory failure patients supported with ECMO. Pre-ECMO data points, demographics, cannulation sites, flow rates, lab values, Vasoactive Ionotropic Score(VIS), P-Prep score, and outcomes were collected and analyzed using t-test and multivariate analyses. We defined a significance as $\mathrm{p}=0.05$.

\section{Results}

35 of 80 ECMO patients were supported for septic shock. Septic patients were larger $(25.1 \mathrm{~kg}$ vs $11.4 \mathrm{~kg}, \mathrm{p}=0.005$ ) and older (85.6 vs 18.8 months, $\mathrm{p}=0.001$ ). Pre-ECMO VIS and P-Prep were both greater in the septic group $(\mathrm{p}=0.007$ and $\mathrm{p}<0.001)$. Pre-ECMO serum lactate level was higher in the septic group ( 3.7 vs $1.4, \mathrm{p}=0.012$ ), but by $96 \mathrm{hrs}$, lactate normalized in both groups. Flow rates at 24 hours were similar between the two groups $(91 \mathrm{~mL} / \mathrm{kg} / \mathrm{min}$ vs $88 \mathrm{~mL} / \mathrm{kg} / \mathrm{min}$, $\mathrm{p}=0.079$ ). No significant difference in bleeding complications or blood product administration was found, but there was a higher incidence of renal failure in septic patients. Survival in the septic group was similar to the comparison group (51.4\% vs $62.2 \%$, p- 0.37$)$. Hours on ECMO and length of stay were also similar.

\section{Conclusion and Potential Impact}

Despite septic patients appearing more ill prior to ECMO, they had similar mortality, support parameters, and outcomes, showing that septic shock is not a contraindication to peripheral ECMO support in pediatric patients. 